Check for updates

Cite this: RSC Adv., 2020, 10, 38884

\title{
A new approach based on isoindole formation reaction for sensitive fluorimetric assay of milnacipran in tablets and biological fluids (plasma/ urine)
}

\begin{abstract}
Ahmed A. Abu-hassan, (D) *a Ramadan Ali $^{a}$ and Sayed M. Derayea ${ }^{b}$
The current study describes a new, sensitive, and economic protocol for milnacipran analysis. Milnacipran was introduced as a therapy for fibromyalgia and depression. It acts by unique and equal inhibition of noradrenaline and serotonin neurotransmitters reuptake. In the presence of 2-mercaptoethanol, the amino moiety of milnacipran condenses with 0 -phthalaldehyde to generate isoindole fluorescent derivative. The isoindole product was measured at $\left(\lambda_{\text {ex }} 338.5 \mathrm{~nm}, \lambda_{\mathrm{em}} 433.5 \mathrm{~nm}\right.$ ) and condensation variables were strictly optimized. The fluorescence intensity of measurements was plotted versus milnacipran concentration to give a linearity range over $200-4000 \mathrm{ng} \mathrm{mL}^{-1}$. The proposed approach was fully validated by the directives of $\mathrm{ICH}$ guidelines and applied without any influence of combined excipient for milnacipran tablet analysis. Furthermore, the procedure was applied in spiked urine and plasma analysis with excellent percentage recovery.
\end{abstract}

Received 12th June 2020

Accepted 14th October 2020

DOI: 10.1039/d0ra05162d

rsc.li/rsc-advances

The approach target was simple development for accurate

\section{Introduction}

Among medical problems that affect many people are fibromyalgia and depression. Fibromyalgia is a complicated disease characterized by many symptoms as generalized chronic muscular pain, sleep disturbance, anxiety, fatigue, cognitive dysfunction, and stiffness. ${ }^{1}$ About $2-4 \%$ of people are affected by fibromyalgia. ${ }^{2}$ Depression is a mood condition that is substantially different from normal mood changes in daily life. Many symptoms are accompanied by depression as weight loss, psychomotor retardation, insomnia, concentration problems, fatigue, and anxiety. In severe states, patients may have a feeling of worthlessness, hallucinations, and thoughts of suicide. ${ }^{3}$ Milnacipran (MLC; 2-(aminomethyl)- $N, N$-diethyl-1phenylcyclopropane-1-carboxamide) was accepted by the FDA as a therapy for depression and fibromyalgia. ${ }^{4}$ It acts by unique and equal inhibition of noradrenaline and serotonin neurotransmitters reuptake. ${ }^{5}$

In the previous analytical literature, MLC was assayed by various techniques which include GC-MS, ${ }^{6}$ liquid chromatography with fluorescence or UV detection, ${ }^{7,8}$ spectrofluorimetry by derivatization with ninhydrin ${ }^{9}$ or $\mathrm{NBD}^{\mathrm{C}} \mathrm{Cl},{ }^{10}$ UVspectrophotometric detection at $223 \mathrm{~nm}^{11,12}$ and colorimetry using ninhydrin ${ }^{13}$ or ion pair complex. ${ }^{14}$

${ }^{a}$ Department of Pharmaceutical Analytical Chemistry, Faculty of Pharmacy, Al-Azhar University, Assiut Branch, Assiut 71524, Egypt. E-mail: ahmedabuhassan@azhar. edu.eg

${ }^{b}$ Department of Analytical Chemistry, Faculty of Pharmacy, Minia University, Minia 61519, Egypt assay of MLC in the spiked biological fluid, and tablets. The method provides advantages over the published method as the heating step is absent which make the method is more facile than the reported methods, ${ }^{9,10}$ non-extractive, ${ }^{13,14}$ low LOD and LOQ, ${ }^{9,11,13,14}$ and finally implemented in non-sophisticated and easily available equipment.

The assay strategy simply relies on isoindole formation reaction. At suitable basic $\mathrm{pH}$, isoindole reaction is the fundamental concept of many derivative reactions used in spectrofluorimetric research. ${ }^{15-18}$ The approach is considered an alternative way for MLC analysis with many advantages. The directives that followed for application and validation were ICH guidelines.

\section{Experimental}

\section{Apparatus}

Throughout the work, fluorescence intensities were monitored by a spectrofluorimeter FS-2 (Scinco, Korea). The instrument is equipped with Xe-arc lamp $(150 \mathrm{~W})$. The voltage of PMT and both monochromators (excitation and emission) slit width and were set at $400 \mathrm{~V}$ and $5 \mathrm{~nm}$ respectively.

\section{Chemicals and materials}

We have been supplied with Milnacipran hydrochloride from Mash Premiere (Badr City, Egypt) pharmaceutical company as a gift. Milnavella (Mash Premiere) and Averomilan ${ }^{\circledR}$ (Averroespharma, Egypt) with $50 \mathrm{mg}$ active milnacipran were utilized in 
dosage form analysis. $o$-Phthalaldehyde (Alpha Chemika, India) was prepared freshly by dissolving $10 \mathrm{mg}$ in $5 \mathrm{~mL}$ methanol and volume adjusted by water to $100 \mathrm{~mL}$ to give concentration $7.46 \times 10^{-4}$ M. 2-Mercaptoethanol (99\%) was purchased from Sigma (MO, USA), stored at $2-8{ }^{\circ} \mathrm{C}$ and diluted by water to give $(0.01 \% \mathrm{v} / \mathrm{v})$ solution. Organic solvent, boric acid, and sodium hydroxide were obtained from El-Nasr chemical-Co (Cairo, Egypt). Borate buffer $(0.2 \mathrm{M})$ solution was prepared by combining suitable volumes of $0.2 \mathrm{M} \mathrm{NaOH}$ and $0.2 \mathrm{M}$ boric acid and adjusting the intended $\mathrm{pH}$ by $\mathrm{pH}$ meter. Human plasma and urine samples were obtained from healthy male volunteers with ages ranged from 30-39 years old at Assiut University Clinics according to institutional guidelines. In all cases, informed written consent was obtained from all participants and plasma samples were kept frozen at $-20{ }^{\circ} \mathrm{C}$ until assessment after gentle thawing. Urine samples were stored in the refrigerator at $4{ }^{\circ} \mathrm{C}$ until analysis.

\section{MLC standard solution}

Stock MLC solution was prepared by dissolving $20 \mathrm{mg}$ of MLC powder in a $100 \mathrm{~mL}$ flask. The powder was initially dissolved in $25 \mathrm{~mL}$ distilled water then completed to the mark with the same solvent. Different MLC working solutions were prepared by further dilution of the previously prepared stock solution.

\section{General analytical procedure}

The procedure was implemented in $10 \mathrm{~mL}$ flask. To each flask $1.4 \mathrm{~mL}$ borate buffer $(\mathrm{pH}=10.5)$ was mixed with an aliquot volume of MLC equal to (200-4000 ng) in the final working solution. The content was thoroughly mixed with $1.2 \mathrm{~mL}$ of 2 mercaptoethanol $(0.01 \mathrm{v} / \mathrm{v})$ followed by $1 \mathrm{~mL} o$-phthalaldehyde $\left(7.46 \times 10^{-4} \mathrm{M}\right)$, then allowed to stand 25 minutes. Finally, the final volume was adjusted with methanol and the fluorescence intensity of the isoindole product was recorded at $\left(\lambda_{\mathrm{ex}} 338.5 \mathrm{~nm}\right.$, $\left.\lambda_{\text {em }} 433.5 \mathrm{~nm}\right)$. All fluorescence reading was corrected against an analytical blank prepared simultaneously.

\section{Tablet formulation analysis}

Ten tablets of Milnavella or Averomilan ${ }^{\circledR}$ were weighed and crushed entirely to a fine powder. A quantity of dosage form powder equal to $50 \mathrm{mg}$ MLC was placed and sonicated in the flask with $30 \mathrm{~mL}$ distilled water for $15 \mathrm{~min}$. The content of the flask was accurately filtered into another volumetric flask and completed to the mark by water. After suitable dilution, the percentage recovery of tablets content was estimated from the regression equation after analysis by the recommended approach procedure.

\section{Steps for spiked human plasma}

At Minia University Hospital, the process of plasma isolation was implemented. The blood was taken from healthy volunteers (who not taken MLC) via the forearm vein, then placed in heparinized tubes. Plasma isolation was accomplished by centrifugation of the tube content at $5000 \mathrm{rpm}$ for $20 \mathrm{~min}$. Until the time of the assay, the plasma was held at $-20{ }^{\circ} \mathrm{C} .{ }^{19}$ Into a series of Eppendorf tubes $(5 \mathrm{~mL}) 800 \mu \mathrm{L}$ of the stored plasma and $200 \mu \mathrm{L}$ of MLC (containing the suitable concentration) was mixed by the vortex. Separation of plasma protein was performed by adding $3 \mathrm{~mL}$ of acetonitrile followed by centrifugation at the time and value mentioned above. Ultimately, $1.0 \mathrm{~mL}$ from the tube content was transferred into a $10 \mathrm{~mL}$ analytical flask and was analyzed by the recommended procedure and the response was corrected against plasma blank.

\section{MLC assay in spiked urine}

Into $50 \mathrm{~mL}$ separating funnel, $800 \mu \mathrm{L}$ human urine, $200 \mu \mathrm{L}$ MLC solution (containing the different concentrations), and $3 \mathrm{~mL}$ $\mathrm{NaOH} /$ carbonate solution $(\mathrm{pH}=11.8)$ was added. Then the solution was extracted three times using $5 \mathrm{~mL}$ dichloromethane in each one. The collected organic layer was carefully evaporated under nitrogen. The MLC residue was reconstituted in methanol. Finally, the assay procedure was followed using a portion of the final methanolic solution and reading corrected against urine blank reading.

\section{Result and discussion}

Several analytical approaches rely on derivatizing a primary amino group by the fluorogenic reagent. Ninhydrin, NBD-Cl, NQS (1,2-naphthoquinone-4-sulfonate), acetylacetone, fluorescamine, and $o$-phthalaldehyde are the prevalent reagents probe. $o$-Phthalaldehyde is more preferred over other fluorogenic reagents due to the absence of the extraction step as in NQS and no heating step like that in ninhydrin, NBD-Cl, NQS, and acetylacetone. Moreover, the reagent has a low price compared to fluorescamine. Although the price of both NBD-Cl and $o$-phthalaldehyde are relatively similar, a very low concentration is needed in the case of $o$-phthalaldehyde $(0.01 \%)$ compared to NBD-Cl $(0.1 \%)$ which would efficiently reduce the cost per run. It is known that heating and extraction steps render analytical assay tedious, and lengthy.

In the presence of a thiol-containing compound as 2-mercaptoethanol, the amino moiety of MLC condenses with $o^{-}$ phthalaldehyde to generate isoindole fluorescent derivative.

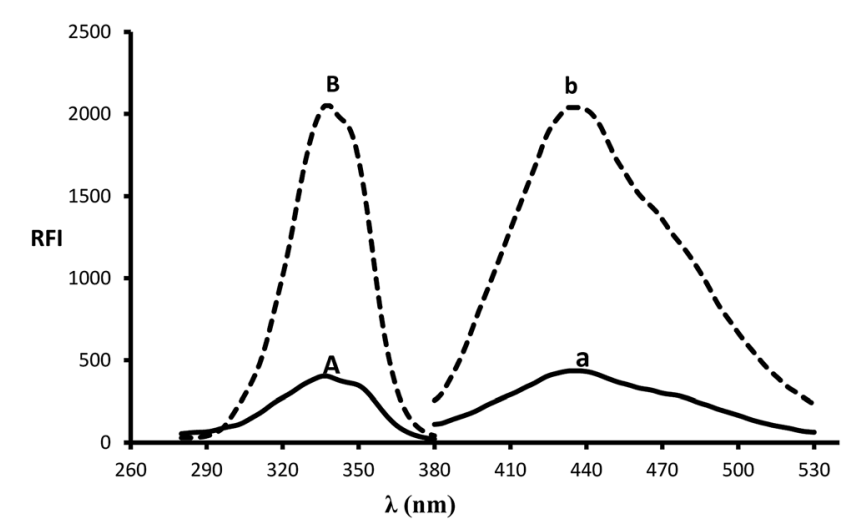

Fig. 1 Excitation and emission spectra ( $B$ and $b$ ) of isoindole product formed from the reaction of MLC (2000 ng mL ${ }^{-1}$ ) with o-phthalaldehyde, in addition to blank solution ( $\mathrm{A}$ and $\mathrm{a}$ ). 
<smiles>CCN(CC)C(=O)C1(c2ccccc2)CC1CN</smiles>

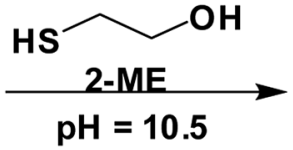

OPA<smiles>CCN(CC)C(=O)C1(c2ccccc2)CC1CN1C(=O)c2ccccc2C1SCCO</smiles>

isoindole fluorescent derivative

Scheme 1 The pathway for isoindole derivative formation between MLC and o-phthalaldehyde/2-mercaptoethanol reagent.

The reaction proceeded in basic $\mathrm{pH}$ and 2-mercaptoethanol is essential for isoindole product stability. ${ }^{20}$ The MLC derivative was monitored at ( $\left.\lambda_{\mathrm{ex}} 338.5 \mathrm{~nm}, \lambda_{\mathrm{em}} 433.5 \mathrm{~nm}\right)$ (Fig. 1) and (Scheme 1) elucidate isoindole formation mechanism. Successful application of the approach on MLC containing dosage forms and biological fluids (plasma/urine) was established after complete validation of the method.

\section{Reaction variables optimization}

The isoindole product was inspected at a variety of wavelengths to choose the accurate wavelength (excitation, emission). Furthermore, variables that affect stability and product formation were precisely studied and optimized.

\section{pH and borate buffer volume}

Inspection of the fluorescence strength of isoindole product at a variety of $\mathrm{pH}$ solution (8-12) was carried out by using borate buffer. Isoindole formation proceeded at alkaline $\mathrm{pH}$ and maximum strength attained at $\mathrm{pH}=10.5$ (Fig. 2). Furthermore, the borate buffer volume was studied by performing several experiments by different volumes $(0.2-2.2 \mathrm{~mL})$. The data of analysis represented in (Fig. 2) reveal that $1.4 \mathrm{~mL}$ is optimum.

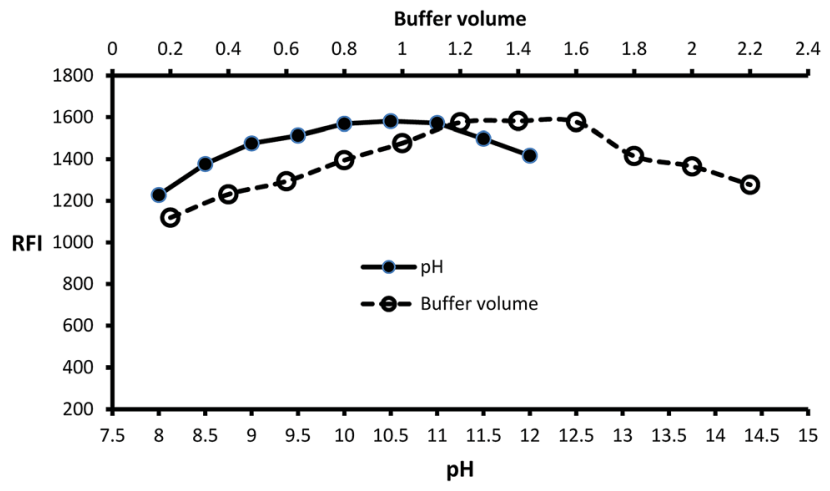

Fig. 2 Effect of $\mathrm{pH}$ and buffer volume on the fluorescence strength of the isoindole product formed from MLC (2000 $\mathrm{ng} \mathrm{mL}^{-1}$ ) with ophthalaldehyde.

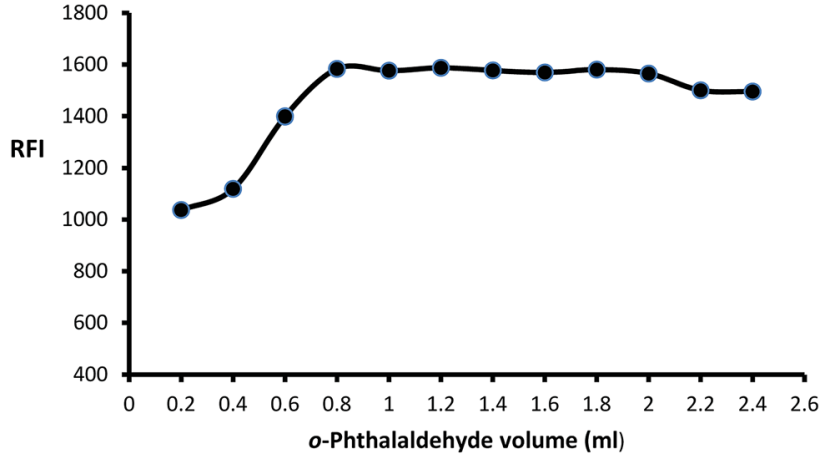

Fig. 3 Effect of different volumes of 0 -phthalaldehyde $\left(7.46 \times 10^{-4}\right.$ $\mathrm{M})$ on the fluorescence intensity of isoindole product using (2000 $\mathrm{ng} \mathrm{mL}^{-1}$ ) of MLC.

\section{Reagent volume (o-phthalaldehyde/2-mercaptoethanol)}

The impact of $o$-phthalaldehyde $\left(7.46 \times 10^{-4} \mathrm{M}\right)$ on the derivative's fluorescence was evaluated by using different volumes $(0.2-2.4 \mathrm{~mL})$. It was noted that FI gradually increased with increasing volume to give constant fluorescence $(0.8-2 \mathrm{~mL})$ followed by a slight gradual decrease (Fig. 3). To stabilize isoindole product, 2-mercaptoethanol addition is an important

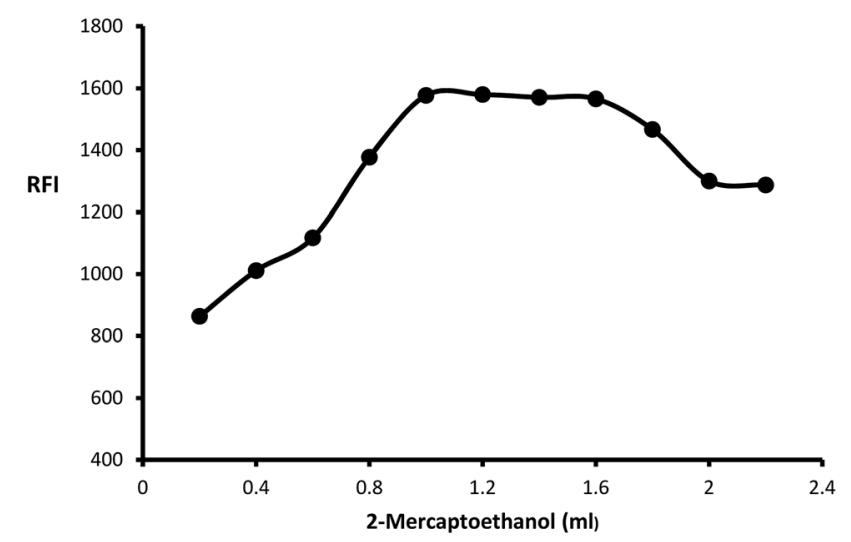

Fig. 4 Effect of different volumes of 2-mercaptoethanol $(0.01 \mathrm{v} / \mathrm{v})$ on the fluorescence intensity of isoindole product using (2000 $\mathrm{ng} \mathrm{mL}^{-1}$ ) of MLC. 


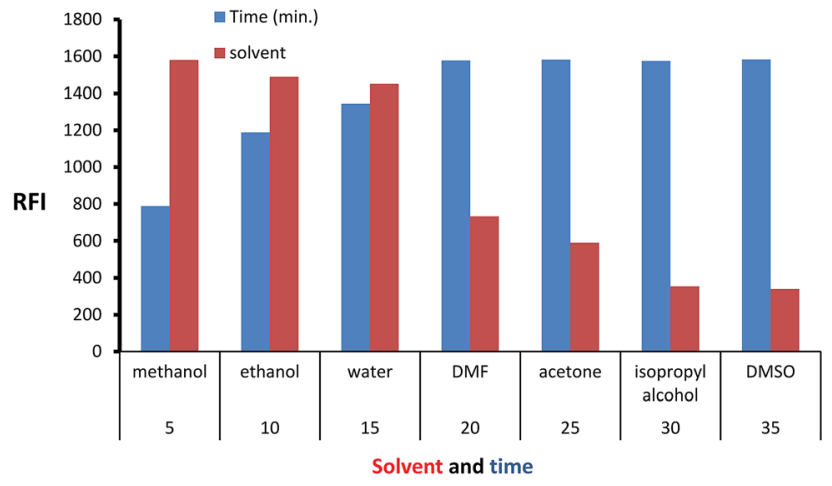

Fig. 5 Effect of time and diluting solvents on the fluorescence intensity of isoindole product using (2000 $\mathrm{ng} \mathrm{mL}^{-1}$ ) of MLC.

step, so the fluorescence strength values were recorded after adding different volumes of 2-mercaptoethanol $(0.01 \mathrm{v} / \mathrm{v})$. The high product fluorescence was recorded by adding $1.2 \pm 0.2$ (Fig. 4).

\section{Time and diluting solvents effect}

Isoindole derivative formed by the reaction of MLC with $o$ phthalaldehyde was diluted by a variety of solvent to select the appropriate one. The inspected solvents were isopropyl alcohol, water, DMSO, methanol, acetone, dimethylformamide, and ethanol (Fig. 5) exhibit high fluorescence value with methanol. The isoindole derivative was allowed to be formed at various time intervals (5-35) minutes. The values of (Fig. 5) show that 25 minutes is adequate time for product formation (isoindole derivative) with maximum FI. Finally, the effect of temperature was tested at different values and the ambient temperature was suitable for isoindole formation.

\section{Work validation}

The procedure of the assay was strictly validated by following directives of ICH guidelines. ${ }^{21}$ The inspected parameters were linearity range, sensitivity (LOQ, LOD), precision, accuracy, and robustness.

Table 1 Regression parameters for the proposed spectrofluorimetric method for the assay of MLC

\begin{tabular}{ll}
\hline Parameter & $\begin{array}{l}\text { Spectrofluorimetric } \\
\text { method }\end{array}$ \\
\hline Linear range $(\mathrm{ng} \mathrm{mL}-1)$ & $200-4000$ \\
Slope & 0.7272 \\
SD of slope $\left(S_{\mathrm{b}}\right)$ & 0.005 \\
Intercept & 133.26 \\
SD of intercept $\left(S_{\mathrm{a}}\right)$ & 10.94 \\
Correlation coefficient $(r)$ & 0.9998 \\
Determination coefficients $\left(r^{2}\right)$ & 0.9997 \\
Number of determinations & 7 \\
Limit of quantitation $\left(\mathrm{ng} \mathrm{mL} \mathrm{mL}^{-1}\right)$ & 150.4 \\
Limit of detection $\left(\mathrm{ng} \mathrm{mL}^{-1}\right)$ & 49.6
\end{tabular}

Table 2 Assessment of the accuracy of the validated method at five concentration levels within the linear range

\begin{tabular}{lccl}
\hline $\begin{array}{l}\text { Taken conc. } \\
\left(\mathrm{ng} \mathrm{mL}^{-1}\right)\end{array}$ & $\begin{array}{l}\text { Found conc. } \\
\left(\mathrm{ng} \mathrm{mL}^{-1}\right)\end{array}$ & Recovery $^{a} \pm \mathrm{SD}$ & \% Error \\
\hline 400 & 393.39 & $98.35 \pm 1.39$ & -1.65 \\
1200 & 1214.35 & $101.20 \pm 1.74$ & 1.20 \\
1800 & 1789.61 & $99.42 \pm 0.35$ & -0.58 \\
2200 & 2214.07 & $100.64 \pm 0.67$ & 0.64 \\
3200 & 3164.29 & $98.88 \pm 0.93$ & -1.12 \\
\multicolumn{4}{l}{} \\
\end{tabular}

Table 3 Intra-day and inter-day precision of the proposed spectrofluorimetric method

\begin{tabular}{|c|c|c|c|c|}
\hline \multirow{2}{*}{$\begin{array}{l}\text { Conc. level } \\
\left(\mathrm{ng} \mathrm{mL}^{-1}\right)\end{array}$} & \multicolumn{2}{|c|}{ Intraday precision } & \multicolumn{2}{|c|}{ Interday precision } \\
\hline & Recovery $^{a} \pm \mathrm{SD}$ & RSD & Recovery $^{a} \pm \mathrm{SD}$ & RSD \\
\hline 400 & $100.64 \pm 1.82$ & 1.81 & $98.46 \pm 1.21$ & 1.23 \\
\hline 1000 & $98.33 \pm 1.73$ & 1.76 & $98.93 \pm 1.20$ & 1.21 \\
\hline 2000 & $100.37 \pm 1.73$ & 1.72 & $101.44 \pm 1.59$ & 1.57 \\
\hline 3000 & $99.38 \pm 0.55$ & 0.56 & $100.37 \pm 0.65$ & 0.64 \\
\hline 3500 & $100.98 \pm 1.77$ & 1.75 & $101.24 \pm 0.85$ & 0.84 \\
\hline
\end{tabular}

${ }^{a}$ Mean of three determinations and SD and RSD are the standard deviation and relative standard deviation respectively.

\section{Linearity range and sensitivity}

Construction of the calibration graph was established at seven standard MLC concentrations. Each MLC concentration was analyzed 3 times by the approach procedure. The fluorescence intensities values were plotted against MLC concentration to derive regression equation. The value of correlation coefficient (0.9998) indicates excellent linearity between response and MLC concentration over the range of (200-4000 $\left.\mathrm{ng} \mathrm{mL} \mathrm{mL}^{-1}\right)$. The statistical data and regression equation was found in (Table 1). The sensitivity of the proposed approach was assessed by LOQ and LOD which is estimated by using equations of $\mathrm{ICH}$ guidelines. The equations state that $\mathrm{LOQ}=10 \sigma / b$ and $\mathrm{LOD}=3.3 \sigma / b$. It is known that $(\sigma)$ is the intercept (SD) standard deviation while $(b)$ is calibration slope. The calculated values were 150.4 and $49.6 \mathrm{ng} \mathrm{mL}{ }^{-1}$ for LOQ and LOD respectively.

Table 4 Robustness study of the proposed method for determination of MLC in pure form

\begin{tabular}{lcc}
\hline Method parameters & Value & $\%$ Recovery $\pm \mathrm{SD}^{a}$ \\
\hline $\mathrm{pH}$ & 10.7 & $98.25 \pm 1.44$ \\
& 10.3 & $101.13 \pm 0.46$ \\
Buffer volume (mL) & 1.3 & $101.13 \pm 1.55$ \\
& 1.5 & $100.39 \pm 1.28$ \\
$o$-Phthalaldehyde volume & 0.9 & $98.96 \pm 1.57$ \\
(mL) & 1.1 & $100.76 \pm 0.55$ \\
2-Mercaptoethanol (mL) & 1.1 & $99.23 \pm 0.56$ \\
& 1.3 & $98.86 \pm 1.49$
\end{tabular}

${ }^{a}$ Mean of three determinations and SD is the standard deviation. 


\section{Accuracy}

Analysis of five MLC concentrations within the calibration curve was performed by the proposed approach. Each MLC concentration was analyzed 3 times and the results were estimated as percentage recovery and the estimated standard deviation. The data in (Table 2) exhibit excellent harmony between the estimated and actual values which confirm the accuracy of the work procedure.

\section{Precision}

Intra-day precision which represents repeatability and inter-day precision were checked at five MLC concentrations within linearity. While intra-day precision was performed on the same day (triplicate measurement for each concentration), inter-day precision was verified on three consecutive days. After data processing, the calculated RSD values were lower than 2 (Table 3) which in agreement with ICH guidance and prove the procedure precision.

\section{Robustness}

Approach robustness was studied by an intended small variation of the assay parameters. The variables varied were $\mathrm{pH}$, buffer volume, $o$-phthalaldehyde, and 2-mercaptoethanol. The results presented in (Table 4 ) confirm that a small variation of the reaction conditions has no significant change in the results which indicates the robustness of the method.

\section{Application}

\section{Pharmaceutical tablets application}

In the Egyptian market, MLC was formulated in many tablets formulation, among them Milnavella or Averomilan ${ }^{\circledR}$. The proposed method was applied for the determination of MLC in the cited products by following the general assay procedure. A comparison of the obtained results with the reported fluorimetric method $^{9}$ was performed by calculating Student's $t$-test and $F$-test to ensure accuracy and precision. At the confidence limit 95\%, the calculated values were lower than the tabulated values (Table 5). This an evidence of the suitability of the approach in dosage form analysis.

\section{Approach application in spiked urine and plasma}

After $2 \mathrm{~h}$ of MLC $(50 \mathrm{mg})$ tablet administration, the maximum

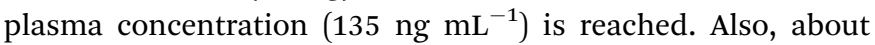

Table 5 Comparison between the proposed and reported method ${ }^{12}$ for the determination of MLC in tablets ${ }^{a}$

\begin{tabular}{lrrrrrr}
\hline & \multicolumn{2}{c}{ Proposed method } & & Reported method $^{12}$ & & \\
Dosage form & Recovery $^{a} \pm \mathrm{SD}$ & & Recovery $^{a} \pm \mathrm{SD}$ & & \multirow{2}{*}{$t$-value $^{\mathrm{a}}$} & $F$-value $^{\mathrm{a}}$ \\
\hline Averomilan $®$ & $98.88 \pm 1.03$ & & $99.34 \pm 0.92$ & 0.74 & 1.27 \\
Milnavella & $101.15 \pm 1.53$ & & $100.62 \pm 0.88$ & 0.67 & 3.04
\end{tabular}

${ }^{a}$ Average of 5 determinations, a; the tabulated $t$ - and $F$-values at the $95 \%$ confidence limit are 2.78 and 6.39 , respectively.
Table 6 Analysis of milnacipran hydrochloride in spiked human urine and plasma using isoindole formation reaction

\begin{tabular}{|c|c|c|c|c|}
\hline \multirow{2}{*}{$\begin{array}{l}\text { Conc. } \\
\left(\mathrm{ng} \mathrm{mL}^{-1}\right)\end{array}$} & \multicolumn{2}{|l|}{ Spiked plasma } & \multicolumn{2}{|l|}{ Spiked urine } \\
\hline & Recovery $^{a} \pm \mathrm{SD}$ & \% Error & Recovery $^{a} \pm \mathrm{SD}$ & \% Error \\
\hline 250 & $101.65 \pm 1.79$ & 1.65 & $101.58 \pm 1.43$ & 1.58 \\
\hline 1000 & $98.17 \pm 1.57$ & -1.83 & $98.26 \pm 1.83$ & -1.74 \\
\hline 1500 & $98.52 \pm 0.71$ & -1.48 & $101.97 \pm 1.04$ & 1.97 \\
\hline 2000 & $101.32 \pm 1.92$ & 1.32 & $98.07 \pm 1.76$ & -1.93 \\
\hline
\end{tabular}

$60 \%$ of MLC excreted in the urine without changing. ${ }^{9}$ Approach application for MLC analysis in spiked (plasma/urine) was established and validated without any notable interference. The data in (Table 6) represent mean recovery of four spiked concentration was estimated from the corresponding regression equation shows the suitability of the approach as a preliminary test for MLC assay in spiked urine and plasma.

\section{Conclusion}

The emphasis in this work was directed in the development of a new, feasible, and sensitive fluorimetric method. The method offers many benefits like simplicity as it implemented in a onepot without heating or extraction, low reagent price, and sensitivity. Furthermore, the measurements were monitored in a spectrofluorometer that is more preferable than other analytical instrumentation. The priority of spectrofluorometer is related to its low price, availability in most laboratories, measurement selectivity, and not require a high expert in usage or tedious sample preparation. The concept of the analytical approach relies on the condensation of MLC amino moiety with $o$-phthalaldehyde to generate isoindole fluorescent derivative in the presence of 2-mercaptoethanol. The isoindole product was monitored at $\left(\lambda_{\text {ex }} 338.5 \mathrm{~nm}, \lambda_{\text {em }} 433.5 \mathrm{~nm}\right)$ and condensation variables were strictly optimized. Finally, the approach applied for MLC analysis in bulk powder and dosage form and involved in urine and spiked plasma analysis with excellent percentage recovery.

\section{Conflicts of interest}

The authors declare that there is no conflict of interest.

\section{References}

1 T. Naresh, S. Shakil S, S. KV, K. Ravi Kiran and K. Suresh, Am. J. Anal. Chem., 2012, 3, 40-49.

2 R. M. Gendreau, M. D. Thorn, J. F. Gendreau, J. D. Kranzler, S. Ribeiro, R. H. Gracely, D. A. Williams, P. J. Mease, S. A. McLean and D. J. Clauw, J. Rheumatol., 2005, 32, 1975-1985.

3 A. T. Beck and B. A. Alford, Depression: Causes and treatment, University of Pennsylvania Press, Philadelphia, 2009. 
4 J. Lopez-Ibor, J. Guelfi, Y. Pletan, A. Tournoux and J. Prost, Int. Clin. Psychopharmacol., 1996, 11, 41-46.

5 M. Briley, J. Prost and C. Moret, Int. Clin. Psychopharmacol., 1996, 11, 9-14.

6 E. Uçaktürk and C. J. C. Şafak, Chromatographia, 2010, 72, 111-119.

7 C. Puozzo, C. Filaquier and G. J. B. Zorza, J. Chromatogr., B, 2004, 806, 221-228.

8 P. B. Parejiya, V. R. Movaliya, B. S. Barot, D. Modi, P. K. Shelat and A. Shukla, J. Liq. Chromatogr. Relat. Technol., 2014, 37, 99-111.

9 I. M. Mostafa, S. M. Derayea, D. M. Nagy and M. A. P. A. M. Omar, Spectrochim. Acta, Part A, 2018, 205, 292-297.

10 I. M. Mostafa, M. A. Omar, D. M. Nagy and S. M. Derayea, RSC Adv., 2018, 8, 22154-22160.

11 N. C. Ratnakar, K. Patel and D. J. Doshi, Asian J. Res. Chem., 2012, 5, 497-499.

12 N. C. Ratnakar, K. Patel and D. J. Doshi, Res. J. Pharm. Technol., 2012, 5, 428-430.
13 M. Mubarakunnisa, A. P. Rani, S. Harika and C. B. Sekaran, Chem. Eng. Sci., 2013, 1, 1-6.

14 V. K. Kumar, V. Srivani, K. V. Prakash, B. M. Ishaq and M. J. Padmalatha, Res. J. Pharm. Technol., 2011, 4, 1250-1251.

15 M. A. Omar, A.-M. I. Mohamed, S. M. Derayea, M. A. Hammad and A. A. J. Mohamed, Spectrochim. Acta, Part A, 2018, 195, 215-222.

16 S. M. Derayea, T. Z. Attia, M. Elnady and D. M. J. Nagy, Microchem. J., 2019, 150, 104143.

17 M. Walash, F. Belal, N. El-Enany and M. El-Maghrabey, Luminescence, 2012, 27, 511-518.

18 S. M. Derayea, T. Z. Attia, M. Elnady and D. M. Nagy, Microchem. J., 2019, 150, 104143.

19 M. A. Abdel-Lateef, M. A. Omar, R. Ali and S. M. Derayea, Spectrochim. Acta, Part A, 2019, 206, 57-64.

20 T. Z. Attia, M. Elnady and S. M. Derayea, $R S C A d v ., 2019,9$, 29942-29948.

21 I. H. T. Guideline, 2005. 